\title{
Split-Hand/Foot Malformation and Subependymal Heterotopia Caused by a DLX5 Gene Mutation: A Case Report
}

\author{
Jun Hee Shin, MD', Min Jeong Han, MD', Sun Jun Kim, MD²,2,3 \\ ${ }^{1}$ Department of Pediatrics, Jeonbuk National University Hospital, Jeonbuk National University Medical School, Jeonju, Korea \\ ${ }^{2}$ Research Institute of Clinical Medicine of Jeonbuk National University, Jeonju, Korea \\ ${ }^{3}$ Biomedical Research Institute of Jeonbuk National University Hospital, Jeonbuk National University Medical School, Jeonju, Korea
}

Received: July 12, 2021

Revised: July 23, 2021

Accepted: July 24, 2021

Corresponding author:

Sun Jun Kim, MD

Department of Pediatrics, Jeonbuk National University Children's

Hospital, 20 Geonji-ro, Deokjin-gu, Jeonju 54907, Korea

Tel: +82-63-250-1799

Fax: +82-63-250-1464

E-mail:sunjun@jbnu.ac.kr
Split hand/foot malformation (SHFM) is a rare congenital malformation involving a limb anomaly [1]. It is characterized by the absence of the central digits of the hand or foot, and is also known as cleft hand or ectrodactyly [2]. SHFM generally an autosomal dominant $(\mathrm{AD})$ condition associated with seven chromosomal loci (SHFM1 to SHFM6 and SHFM with long bone deficiency [SHFLD] ) [3]. Involvement Dlx- distalless-related homebox 5 (DLXS) gene expression in the SHFM1 type has been reported with autosomal recessive (AR) inheritance in a family; furthermore, SHFM6 also shows an AR inheritance pattern and SHFM2 is an X-linked recessive condition [4]. DLX5 and DLX6 are expressed in the embryonic limb buds and ectoderm, are necessary for craniofacial, axial, and appendicular skeleton development, and also promote cortical interneuron migration from the basal forebrain [5-8]. Herein, we report the first case of a male SHFM patient with deafness and subependymal heterotopia that appeared to be inherited as a heterogeneous condition of the DLX5 gene.

A 1-day-old male patient was admitted to our Department of Pediatrics for evaluation and management of ectrodactyly detected on prena- tal ultrasonography. The patient was the parents' first baby and was born via an emergency cesarean section due to premature rupture of membranes at 39 weeks of gestation. At birth, the weight was $3,960 \mathrm{~g}$ (>90th percentile), the height was $47.3 \mathrm{~cm}$ (10th-25th percentile), and the head circumference was $35.1 \mathrm{~cm}$ (75th-90th percentile). Ectrodactyly of both hands and feet were observed and there were no other abnormal findings on an infantogram (Fig. 1). His father also had ectrodactyly on both hands and feet, as well as bilateral congenital deafness, and was unable to communicate verbally. The patient's father was the fourth of six siblings, but no one in his family had similar anomalies. Additionally, the patient's father did not have a history of convulsions.

Subependymal nodules on the right occipital horn were identified on brain magnetic resonance imaging that was performed at 7 months of age due to an afebrile seizure (Fig. 2). He received a cochlear implant after the confirmation of hearing impairment in an auditory brainstem response test (hearing threshold more than 90 $d B)$. At the age of 5, the patient presented to our emergency room due to a 2-minute afebrile focal seizure. Electroencephalography showed asym-

Copyright (C) 2021 Korean Child Neurology Society

This is an Open Access article distributed under the terms of the Creative Commons Attribution Non-Commercial License (http://creativecommons.org/licenses/by-nc/4.0/) which permits unrestricted non-commercial use, distribution, and reproduction in any medium, provided the original work is properly cited. 

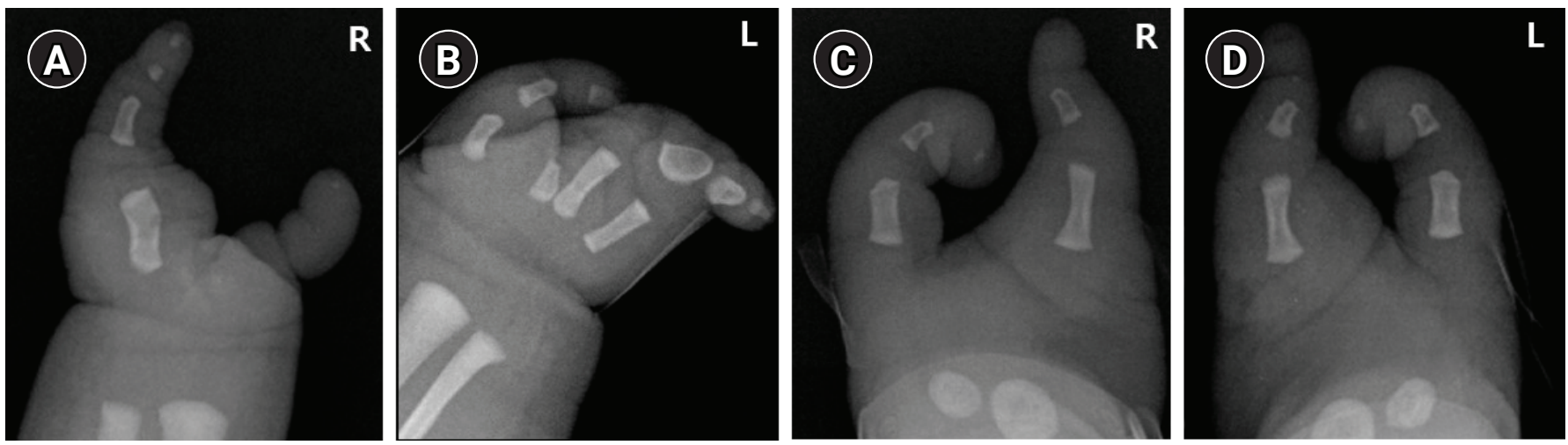

Fig. 1. Radiological images of the hands and feet showing complete absence of the second, third, and fourth digits on both $(A, B)$ hands and $(C, D)$ feet.
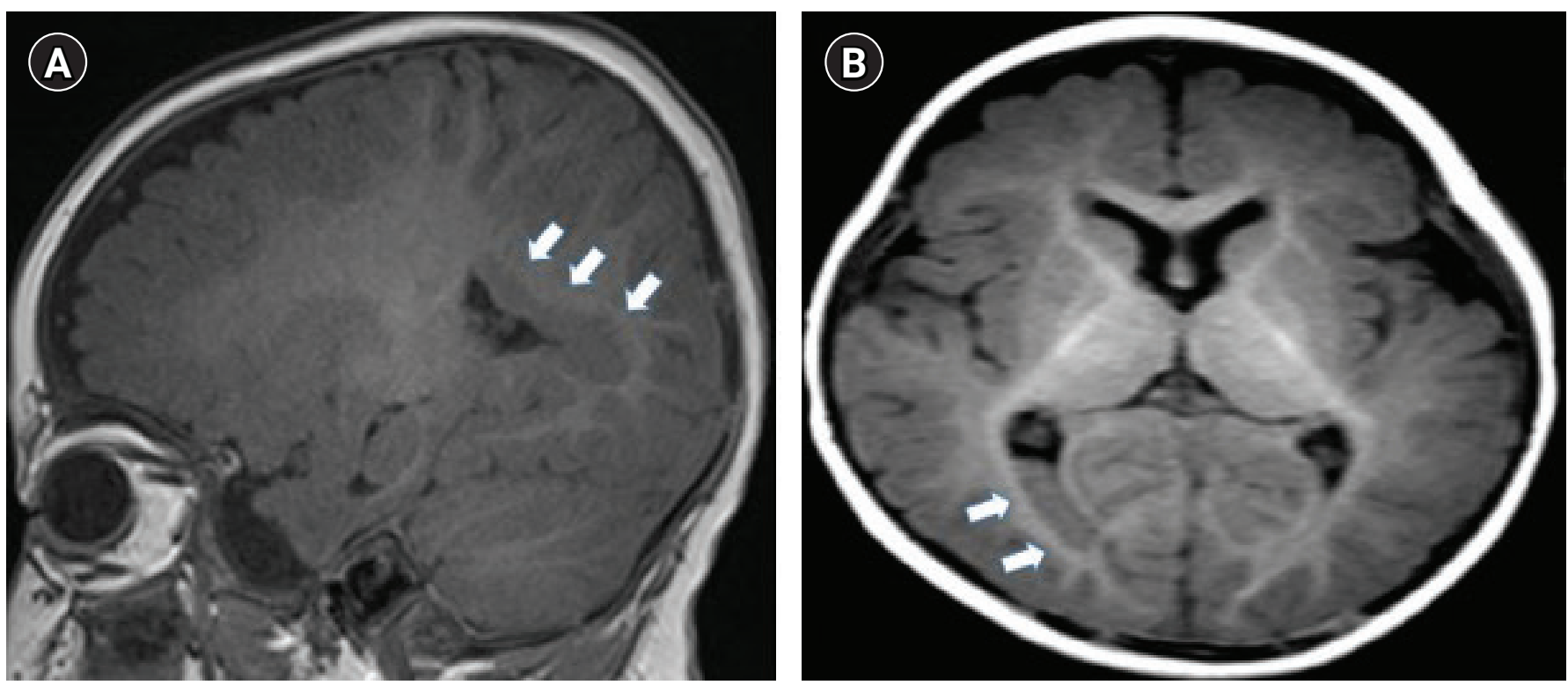

Fig. 2. Brain magnetic resonance imaging. (A) A T1-weighted sagittal image and (B) T2-weighted axial image show subependymal heterotopia on the right occipital horn (white arrows).

metric background activity due to depressed background activity in the right mid-posterior area and sharp waves in both frontocentral areas. The patient was diagnosed with focal epilepsy and began treatment with levetiracetam. Genetic testing was performed for the patient, over the objections of his father, who also chose not to receive a genetic evaluation himself. Genetic variants were not detected in the subependymal heterotopia-related periventricular nodular heterotopia (PVNH) 1 and $\mathrm{PVNH} 2$ genes; however, a heterozygous mutation was detected in the SHFM-related DLX5 gene (NM_005221.5:c. 557A > G (p.Gln186Arg, hetero). A mouse-model study conducted in 2002 reported that double knock-out of both genes ( $D l \times 5 / D l \times 6-/-)$ resulted in typical ectrodactyly, with craniofacial and inner ear abnormalities [9]. This case showed a heterozygous DLX5 gene mutation, but the pa- tient-like his father-showed typical phenotypes of DLX5 mutation, including ectrodactyly and craniofacial and inner ear abnormalities, as well as hearing impairment and SHFM. This case has clinical significance because, although we were unable to perform a parental genetic study, the presence of a similar phenotype in the father shows that DLX5 mutation could have had an $\mathrm{AD}$ inheritance pattern. In addition to our case of a DLX5 mutation, a patient with SHFM caused by a novel heterozygous variant in the DLX6 gene, which is also known to show AR inheritance, was reported in 2017 [10]. After cochlear implantation, the patient was diagnosed with grade 2 hearing impairment. He has received speech-language therapy and an anticonvulsant agent. Other areas of development, including motor and cognition, have been well maintained and he is doing well without seizure relapse. 
This study was approved by the Institutional Review Board of Jeonbuk National University Hospital (IRB no: 2021-06-072). Written informed consent was obtained from this patient.

\section{Conflicts of interest}

No potential conflict of interest relevant to this article was reported.

\section{ORCID}

Jun Hee Shin, https: / / orcid.org/0000-0001-6460-2770

Min Jeong Han, https://orcid.org/0000-0002-7058-3657

Sun Jun Kim, https:/ /orcid.org/0000-0002-7673-8728

\section{Author contribution}

Conceptualization: JHS, MJH, and SJK. Data curation: JHS, MJH, and SJK. Methodology: JHS, MJH, and SJK. Project administration: JHS, MJH, and SJK. Visualization: JHS, MJH, and SJK. Writing-original draft: JHS, MJH, and SJK. Writing-review \& editing: JHS, MJH, and SJK.

\section{References}

1. Sowinska-Seidler A, Socha M, Jamsheer A. Split-hand/foot malformation: molecular cause and implications in genetic counseling. J Appl Genet 2014;55:105-15.

2. Moerman P, Fryns JP. Ectodermal dysplasia, Rapp-Hodgkin type in a mother and severe ectrodactyly-ectodermal dysplasia-clefting syndrome (EEC) in her child. Am J Med Genet
1996;63:479-81.

3. Umair M, Hayat A. Nonsyndromic split-hand/foot malformation: recent classification. Mol Syndromol 2020;10:243-54.

4. Shamseldin HE, Faden MA, Alashram W, Alkuraya FS. Identification of a novel DLX5 mutation in a family with autosomal recessive split hand and foot malformation. J Med Genet 2012;49:16-20.

5. Proudfoot A, Axelrod HL, Geralt M, Fletterick RJ, Yumoto F, Deacon AM, et al. Dlx5 homeodomain: DNA complex. Structure, binding and effect of mutations related to split hand and foot malformation syndrome. J Mol Biol 2016;428:1130-41.

6. Restelli M, Lopardo T, Lo Iacono N, Garaffo G, Conte D, Rustighi A, et al. DLX5, FGF8 and the Pin1 isomerase control $\Delta \mathrm{Np} 63$ a protein stability during limb development: a regulatory loop at the basis of the SHFM and EEC congenital malformations. Hum Mol Genet 2014;23:3830-42.

7. Simeone A, Acampora D, Pannese M, D'Esposito M, Stornaiuolo A, Gulisano M, et al. Cloning and characterization of two members of the vertebrate Dlx gene family. Proc Natl Acad Sci U S A 1994;91:2250-4.

8. Levi G, Puche AC, Mantero S, Barbieri O, Trombino S, Paleari $\mathrm{L}$, et al. The Dlx5 homeodomain gene is essential for olfactory development and connectivity in the mouse. Mol Cell Neurosci 2003;22:530-43.

9. Robledo RF, Rajan L, Li X, Lufkin T. The Dlx5 and Dlx6 homeobox genes are essential for craniofacial, axial, and appendicular skeletal development. Genes Dev 2002;16:1089-101.

10. Ullah A, Hammid A, Umair M, Ahmad W. A novel heterozygous intragenic sequence variant in DLX6 probably underlies first case of autosomal dominant split-hand/foot malformation type 1. Mol Syndromol 2017;8:79-84. 